\title{
AUS DEM VORWORT DES VERFASSERS ZUR ERSTEN DEUTSCHEN AUFLAGE
}

Im Frühjahr 1954 hielt ich als Gastprofessor an der Humboldt-Universität zu Berlin Vorlesungen über Wahrscheinlichkeitsrechnung. Zur gleichen Zeit bereitete ich die zweite (russische) Auflage des vorliegenden Lehrbuches vor. Deutsche Kollegen machten mir den Vorschlag, dieses Lehrbuch in die deutsche Sprache zu übersetzen, und das Forschungsinstitut für Mathematik der Deutschen Akademie der Wissenschaften und der Akademie-Verlag haben sich freundlicherweise bereit erklärt, die Herausgabe der Ubersetzung zu besorgen.

Mein Hörer und Schüler Wolfgang Richter - damals Student der HumboldtUniversität - fertigte die Ubersetzung an. Herr HaNs Joachim Rossberg wissenschaftlicher Assistent an der Deutschen Akademie der Wissenschaften übernahm die Redaktionsarbeit. Er führte diese Arbeit mit der größten Sorgfalt durch, überarbeitete den deutschen Text und überprüfte Formeln und rechnerische Ableitungen. Dabei hat er eine Reihe von Rechenfehlern feststellen und beheben können; seine diesbezüglichen Hinweise habe ich auch bei der Herausgabe der zweiten russischen Auflage berücksichtigt. Ich möchte hiermit dem Forschungsinstitut für Mathematik der Deutschen Akademie der Wissenschaften, dem Akademie-Verlag und den genannten Kollegen für ihre Mühe und ihre sorgfältige Arbeit meinen herzlichsten Dank aussprechen.

Das vorliegende Lehrbuch ist nur eine elementare Einleitung in die Wahrscheinlichkeitsrechnung. Dies rechtfertigt zum Teil die Tatsache, da $B$ einige wichtige Gebiete (wie z. B. Markowsche Ketten) nur kurz, andere überhaupt nicht behandelt werden. Andererseits glaube ich, da $B$ es für den angehenden Mathematiker wichtig ist, Verbindungen zwischen verschiedenen mathematischen Disziplinen und den Zusammenhang zwischen Mathematik und Praxis zu erkennen. Ich habe mich bemüht, diese Seite möglichst klar heraustreten zu lassen. Diesem Zweck dienen u. a. verschiedene im Text behandelte Beispiele und dem Leser empfohlene Aufgaben, von denen viele angewandten Charakter haben. Doch möchte ich gleich bemerken, da $B$ diese Beispiele und Aufgaben hier nur zur Illustration der Theorie dienen und keinen Anspruch erheben, dem Leser konkrete Kenntnisse über Anwendungen der Wahrscheinlichkeitsrechnung in Naturwissenschaften und Technik zu vermitteln. Es kommt noch hinzu, daß wichtige Anwendungsgebiete der Wahrscheinlichkeitsrechnung in dem Buch nicht einmal erwähnt worden sind.

Eingehende Kenntnisse über die in dem Lehrbuch angeschnittenen Gebiete können nur durch das Studium der entsprechenden Zeitschriftenliteratur und 
Monographien erworben werden. Für die Theorie der stochastischen Prozesse möchte ich in erster Linie auf die ausführlichen Bücher von A. BLANC-LAPIERRE und R. Forter und von J.L. Doos sowie auf den ausgezeichneten Artikel von A. M. JAGLOM (zum Kapitel X) hinweisen. Für den Leser, der stärkeres Interesse für angewandte Aufgaben hat und sich z. B. für Probleme der Massenbedienung interessiert, möchte ich das kürzlich in russischer Sprache erschienene Buch von A. J. Chrivsschin ,Mathematische Methoden der Theorie der Massenbedienung" (Trudy Math. Inst. Stekloff 49 (1955)) auf das wärmste empfehlen.

Der kurze historische Abriß am Ende meines Lehrbuches erhebt keinen Anspruch auf Vollständigkeit. Er gibt eine Vorstellung über die Entwicklung der Wahrscheinlichkeitsrechnung in Europa bis zur Mitte des XIX. Jahrhunderts. Eine ausführliche Ubersicht über die moderne Entwicklung der Wahrscheinlichkeitsrechnung ist bis jetzt noch nicht geschrieben worden. Der Verfasser hofft, in den folgenden russischen Auflagen des Buches diese Lücke schließen zu können.

Ich würde mich sehr freuen, wenn die Herausgabe der vorliegenden deutschen Ubersetzung des Lehrbuches dazu beitrüge, das Interesse des deutschen mathematischen Nachwuchses für das Gebiet der Wahrscheinlichkeitsrechnung zu verstärken.

B. GNEDENKo 
